\title{
Interacting errors in large-eddy simulation: a review of recent developments
}

\author{
BERNARD J. GEURTS*
}

Multiscale Modeling and Simulation, NACM, J.M. Burgers Center, Faculty EEMCS, University of Twente, P.O. Box 217, 7500 AE Enschede, The Netherlands, and Anisotropic Turbulence, Fluid Dynamics Laboratory, Department of Applied Physics, Eindhoven University of Technology, P.O. Box 513, 5600 MB Eindhoven, The Netherlands

The accuracy of large-eddy simulations is limited, among others, by the quality of the subgrid parameterisation and the numerical contamination of the smaller retained flow structures. We review the effects of discretisation and modelling errors from two different perspectives. We first show that spatial discretisation induces its own filter and compare the dynamic importance of this numerical filter to the basic large-eddy filter. The spatial discretisation modifies the large-eddy closure problem as is expressed by the difference between the discrete 'numerical stress tensor' and the continuous 'turbulent stress tensor'. This difference consists of a high-pass contribution associated with the specific numerical filter. Several central differencing methods are analysed and the importance of the subgrid resolution is established. Second, we review a database approach to assess the total simulation error and its numerical and modelling contributions. The interaction between the different sources of error is shown to lead to their partial cancellation. From this analysis one may identify an 'optimal refinement strategy' for a given subgrid model, discretisation method and flow conditions, leading to minimal total simulation error at a given computational cost. We provide full detail for homogeneous decaying turbulence in a 'Smagorinsky fluid'. The optimal refinement strategy is compared with the error reduction that arises from grid refinement of the dynamic eddy-viscosity model. The main trends of the optimal refinement strategy as a function of resolution and Reynolds number are found to be adequately followed by the dynamic model. This yields significant error reduction upon grid refinement although at coarse resolutions significant error levels remain. To address this deficiency, a new successive inverse polynomial interpolation procedure is proposed with which the optimal Smagorinsky constant may be efficiently approximated at a given resolution. The computational overhead of this optimisation procedure is shown to be well justified in view of the achieved reduction of the error level relative to the 'no-model' and dynamic model predictions.

\section{Introduction}

Direct numerical simulation and large-eddy simulation are two important strategies for the numerical investigation of turbulent flows. Within the constraints of present-day computing

35 infrastructure, the direct simulation approach is adopted for full resolution of flow problems of modest complexity, e.g., to under-pin theoretical and modelling studies. Instead, the focus in large-eddy simulation is on a computationally more accessible coarsened flow description

This paper is associated with the focus-issue Quality assessment of unsteady methods for turbulent combustion prediction and validation.

${ }^{*}$ Corresponding author. E-mail: b.j.geurts@utwente.nl 
which is obtained by low-pass spatial filtering. This allows an external control over the required spatial resolution. However, low-pass filtering gives rise to the well-known closure problem for the turbulent stress which represents the dynamic effects of the filtered-out small-scale turbulence on the retained flow structures. Viewed entirely from the PDE level corresponding to the spatially filtered Navier-Stokes equations, the remaining task is to close the system of equations by modelling these small-scale dynamic effects in terms of the resolved flow. Considerable effort has been put into construction, testing and tuning of such so-called subgrid models over the past few years [1-4]. In such testing procedures, one, frequently compares predictions from large-eddy simulations to filtered data from direct numerical simulation and/or experimental observations (see also, e.g., [5-7]).

The above sketch of direct and large-eddy simulation is incomplete in at least one important respect as it does not contain the unavoidable subsequent discretisation step. In fact, since the numerical representation is typically associated with only marginal subgrid resolution, a significant alteration of the resolved scales' dynamics may be introduced in the computational model [8-11]. It is the purpose of this paper to review and quantify the numerical error dynamics explicitly for a number of characteristic, well-known discretisation methods. Next to the filter width $\Delta$, the specification of the numerical method implies the introduction of a second length scale $h$ which characterises the (local) computational grid spacing. Correspondingly, the discretisation step induces a second element of possible flow filtering. The difficulty hence resides in assessing the modelling and discretisation errors and their dynamic interaction in order to arrive at a specification of simulation and modelling parameters which are optimal, i.e. yield minimal total simulation error in the quantities of interest, at a given computational cost. In this paper we review consequences of the interactions that occur between discretisation and subgrid modelling errors. Moreover, we propose a simple optimisation procedure to approximate optimal simulation parameters.

The relative importance of the turbulent stresses compared to the numerically induced contributions depends strongly on the sub-filter resolution $r=\Delta / h[12,13]$. If $r$ is sufficiently large, the grid-independent large-eddy solution consistent with the assumed value of $\Delta$ may be accurately approximated. However, large-eddy simulation of applications with a realistic complexity is typically associated with only a marginal resolution corresponding to $r=1-$ 2. In that case, the numerically induced effects are comparable to or even larger than the turbulent stresses for typical discretisation methods such as central or upwind finite difference or finite volume methods. Thereby, the computational large-eddy closure typically contains an important contribution which is sensitive to the adopted spatial discretisation.

Central to a framework for assessing the error behaviour associated with an actual largeeddy implementation is the evaluation of the total simulation error and its decomposition into numerical and modelling components. By appropriately comparing large-eddy and direct simulation predictions, the total simulation error can be quantified [10, 14]. Recently, a database of both direct and large-eddy simulations of decaying homogeneous, isotropic turbulence was analysed at two different Reynolds numbers [11]. In particular, for the Smagorinsky eddyviscosity model combined with second-order finite volume discretisation, the dependence of modelling and numerical errors on simulation parameters was discussed. The interaction between these two basic sources of error was shown to lead to their partial cancellation for several flow properties. The effects of numerical errors in wall-bounded turbulent flows were investigated recently in [15] and the use of explicit filtering to externally control the error dynamics was studied in $[16,17]$.

In this paper we review the interacting error dynamics in terms of a so-called error landscape which provides a concise visualisation of the induced errors [11]. The 'optimal refinement strategy' that yields a minimal total simulation error at a given computational effort may be identified from this error landscape. Compared to the optimal refinement strategy, the error 
induced by the dynamic eddy-viscosity model [18] at different resolutions is about a factor 2 larger. However, the rate by which the error reduces with increased resolution was shown to be quite strong, particularly at high resolutions [19]. To compensate for the remaining high error levels at coarse resolutions, a new successive inverse polynomial interpolation (SIPI) procedure is proposed to efficiently approximate the optimal Smagorinsky constant at a given resolution. As a point of reference, we start from predictions using either no-subgrid model at all or the dynamic eddy-viscosity model at a given spatial resolution. The proposed iterative procedure rapidly converges towards the optimal model parameter. The computational overhead of this procedure is shown to be well justified by the increased accuracy.

The organisation of this paper is as follows. In section 2 we introduce the governing equations and consider the filter that is induced by the spatial discretisation. Attention is focussed on the modification of the large-eddy closure problem, particularly at comparably large filter 100 widths and coarse subgrid resolutions. Section 3 is devoted to a database analysis of largeeddy simulation of homogeneous decaying turbulence in a 'Smagorinsky fluid'. An exhaustive database approach is rather expensive, but allows us to identify the optimal refinement strategy. To achieve more practical error-reduction approaches, the degree of optimality of the dynamic procedure is discussed. Further improvements in accuracy may be achieved by directly ap-

105 proximating the optimal model parameters at a given resolution. A new iterative procedure is suggested and discussed in section 3. Concluding remarks are collected in section 4 .

\section{Governing equations, spatial discretisation and induced filter}

In this section we introduce the governing equations and identify the errors associated with their spatial discretisation and subgrid closure. Moreover, we discuss the properties of the

110 spatial filter that is induced by spatial discretisation and establish its dynamic importance in terms of the subgrid resolution $r=\Delta / h$.

Filtering the Navier-Stokes equations requires a low-pass spatial filter $L$. Often, a convolution filter is adopted which in one spatial dimension associates the filtered velocity $\bar{u}$ with the unfiltered velocity $u$ through

$$
\bar{u}=L(u)=\int_{-\infty}^{\infty} g(x-s, \lambda) u(s) \mathrm{d} s
$$

115 with normalised filter kernel $g(z, \lambda)$. The filter kernel $g$ is characterised by an externally specified length scale $\lambda$ which directly determines the effective filter width $\Delta$ [20] through

$$
\frac{1}{\Delta}=\int_{-\infty}^{\infty} g^{2}(z, \lambda) \mathrm{d} z .
$$

This definition applies to all kernels that are square integrable [1]. Other definitions proposed in the literature (see [4] for an overview) are more restricted in their applicability to different filters.

120 For incompressible fluids, the application of the filter $L$ to the continuity and Navier-Stokes equations leads to

$$
\partial_{j} \bar{u}_{j}=0, \quad \partial_{t} \bar{u}_{i}+\partial_{j}\left(\bar{u}_{j} \bar{u}_{i}\right)+\partial_{i} \bar{p}-\frac{1}{R e} \partial_{j j} \bar{u}_{i}=-\partial_{j}\left(\overline{u_{i} u_{j}}-\bar{u}_{j} \bar{u}_{i}\right)=-\partial_{j} \tau_{i j} .
$$

Here, $\partial_{t}$ (respectively $\partial_{j}$ ) denotes partial differentiation with respect to time $t$ (respectively spatial coordinate $x_{j}$ ). Summation over repeated indices is implied. The component of the filtered velocity in the $x_{j}$ direction is $\bar{u}_{j}$, and $\bar{p}$ is the filtered pressure. Finally, Re denotes the Reynolds number of the flow. In this formulation, the closure problem on the PDE level 
is expressed by the divergence of the turbulent stress tensor $\tau_{i j}={\overline{u_{i}}}_{u_{j}}-\bar{u}_{j} \bar{u}_{i}$. To close the filtered equations, it is quite common in literature to approximate $\tau_{i j}$ by a subgrid model that can be evaluated in terms of operations on the filtered velocity field only. Next to such deterministic subgrid models, other approaches incorporate a stochastic element to represent the dynamic consequences of $\tau_{i j}$ [21-23].

To illustrate the spatial discretisation and subgrid modelling, we follow the numerical treatment of the modelled convective term:

$$
\begin{aligned}
\partial_{j}\left(\overline{u_{i} u_{j}}\right)+\partial_{i} \bar{p} & =\left[\delta_{j}\left(\bar{u}_{i} \bar{u}_{j}\right)+\delta_{i} \bar{p}+\mathcal{D}_{i}\right]+\partial_{j} \tau_{i j} \\
& =\left[\delta_{j}\left(\bar{u}_{i} \bar{u}_{j}\right)+\delta_{i} \bar{p}+\mathcal{D}_{i}\right]+\left[\partial_{j} m_{i j}+\mathcal{R}_{i}\right] \\
& =\delta_{j}\left(\bar{u}_{i} \bar{u}_{j}\right)+\delta_{i} \bar{p}+\delta_{j} m_{i j}+\left[\mathcal{D}_{i}+\mathcal{R}_{i}+\mathcal{D}_{i}^{(m)}\right],
\end{aligned}
$$

where we introduced $\mathcal{D}_{i}=\left[\partial_{j}\left(\bar{u}_{i} \bar{u}_{j}\right)-\delta_{j}\left(\bar{u}_{i} \bar{u}_{j}\right)\right]+\left[\partial_{i} \bar{p}-\delta_{i} \bar{p}\right]$ to measure the discretisation error arising from the discretisation method $\delta_{j}, \mathcal{R}_{i}=\partial_{j}\left(\tau_{i j}-m_{i j}\right)$ to represent the total 'model residue' and $\mathcal{D}_{i}^{(m)}$ to quantify discretisation errors when treating the model $m_{i j}$. The latter type of errors obviously depends on the subgrid model that is used and may include contributions due to the coarse grid implementation of explicit filtering or the evaluation of the velocity-gradient tensor. The total simulation error in any large-eddy simulation arises from the different error contributions $\mathcal{D}_{i}, \mathcal{R}_{i}$ and $\mathcal{D}_{i}^{(m)}$ and their interactions. This forms the focus of this paper.

To provide a specific class of computational large-eddy formulations we consider as a basic subgrid model the classical Smagorinsky [24] model $m_{i j}^{\mathrm{S}}$ to approximately represent $\tau_{i j}$. In particular, this implies

$$
\tau_{i j} \rightarrow m_{i j}^{S}=-2\left(C_{\mathrm{S}} \Delta\right)^{2}|\bar{S}| \bar{S}_{i j}=-2 \ell_{S}^{2}|\bar{S}| \bar{S}_{i j}
$$

where $|\bar{S}|=\left(2 \bar{S}_{i j} \bar{S}_{i j}\right)^{1 / 2}$ is the magnitude of the filtered rate of the strain tensor $\bar{S}_{i j}=\partial_{i} \bar{u}_{j}+$ $\partial_{j} \bar{u}_{i}$, and we introduced the Smagorinsky length $\ell_{\mathrm{S}}=C_{\mathrm{S}} \Delta$ for later convenience. We will not adopt a specific value for $C_{\mathrm{S}}$ or $\Delta$, but rather consider the product $\ell_{\mathrm{S}}=C_{\mathrm{S}} \Delta$ as the relevant parameter [25].

Next to the standard Smagorinsky model, we will also consider the dynamic procedure in which the eddy-viscosity coefficient is determined self-consistently [26, 27]. To introduce the dynamic model, we require next to the basic large-eddy filter $\overline{(\cdot)}$ an explicit test filter $\widetilde{(\cdot)}$. Associated with these two filters, two different turbulent stress tensors arise, i.e. $\tau_{i j}=\overline{u_{i} u_{j}}-$ $\bar{u}_{i} \bar{u}_{j}$ corresponding to the large-eddy filter and $T_{i j}=\widetilde{u_{i} u_{j}}-\widetilde{\bar{u}}_{i} \widetilde{\bar{u}}_{j}$ based on the composition of the test filter and the basic large-eddy filter. The central identity obeyed by these stress tensors may be expressed as [26]

$$
T_{i j}-\widetilde{\tau}_{i j}=\mathcal{L}_{i j}, \quad \mathcal{L}_{i j}={\widetilde{\bar{u}_{i}}}_{j}-\widetilde{\bar{u}}_{i} \widetilde{\bar{u}}_{j}
$$

Here, we introduced the resolved stress tensor $\mathcal{L}_{i j}$ which is available during a large-eddy 155 simulation.

The standard dynamic procedure proceeds by assuming that $\tau_{i j}$ and $T_{i j}$ can be approximated with the same model, i.e. $\tau_{i j} \rightarrow m_{i j}(\overline{\mathbf{u}})$ and $T_{i j} \rightarrow m_{i j}(\tilde{\overline{\mathbf{u}}})$. In line with the basic Smagorinsky eddy-viscosity model, we put $m_{i j}(\overline{\mathbf{u}})=-2 \ell_{d}^{2}|\bar{S}| \bar{S}_{i j}$ and introduce the filter ratio $\alpha=\widetilde{\Delta} / \Delta$ and the dynamic length scale $\ell_{d}=\Delta \sqrt{C_{d}}$. Within the usual least-squares approach [27], one obtains

$$
\ell_{d}^{2}=C_{d} \Delta^{2}=\frac{1}{2} \frac{\left\langle\mathcal{L}_{i j} M_{i j}\right\rangle}{\left\langle M_{i j} M_{i j}\right\rangle}, \quad M_{i j}=\left|\widetilde{\bar{S} \mid \bar{S}_{i j}}-\alpha^{2}\right| \widetilde{\bar{S}} \mid \widetilde{\bar{S}}_{i j}
$$

In (7) we denote averaging over the flow domain by $\langle\cdot\rangle$. We consider the top-hat filter as a test filter with $\widetilde{\Delta}=\alpha h$ and use $\alpha=2$ [28]. The dependence on $\alpha$ was discussed in [11]. 
The governing equations are discretised using the so-called method of lines. The time165 stepping method which we adopt is an explicit four-stage compact-storage Runge-Kutta method [1]. We will use a second order finite volume method for the convective, viscous and subgrid fluxes [14].

The discretisation of the governing equations introduces a separate source of approximation in a large-eddy simulation. Therefore, the numerical method should be fully included in the 170 analysis [29]. We start from the discrete convective flux $\delta_{x}\left(\bar{u}^{2}\right)$ that is directly available in the computational model and group the remainder into the closure problem. We recall that the discrete derivative operator can be expressed as $\delta_{x} f=\partial_{x} \widehat{f}$ where $\widehat{f}$ is used here to denote a numerically filtered variable [8]. Restricting ourselves to this representation, we have for the model of the 1D Burgers equation

$$
\begin{aligned}
\partial_{t} \bar{u}+\frac{1}{2} \partial_{x}\left(\overline{u^{2}}\right) & =\partial_{t} \bar{u}+\frac{1}{2} \delta_{x}\left(\bar{u}^{2}\right)+\frac{1}{2}\left[\partial_{x}\left(\overline{u^{2}}\right)-\delta_{x}\left(\bar{u}^{2}\right)\right] \\
& =\partial_{t} \bar{u}+\frac{1}{2} \partial_{x}\left(\widehat{\bar{u}}^{2}\right)+\frac{1}{2} \partial_{x}(\Xi)=0,
\end{aligned}
$$

175 in which we introduced the numerical turbulent stress tensor

$$
\Xi=\overline{u^{2}}-\widehat{\bar{u}^{2}}=\left(\overline{u^{2}}-\bar{u}^{2}\right)+\left(\bar{u}^{2}-\widehat{\bar{u}}^{2}\right)=\tau+\left(\bar{u}^{2}-\widehat{\bar{u}^{2}}\right)=\tau+\mathcal{H}^{(\uparrow)}\left(\bar{u}^{2}\right),
$$

where $\mathcal{H}^{(\urcorner)}(f)=f-\widehat{f}$ denotes a high-pass filter associated with $\widehat{(\cdot)}$. We observe that the difference between $\Xi$ and $\tau$ may be written in terms of a high-pass filter applied to $\bar{u}^{2}$. Consistent with this decomposition, subgrid modelling of $\Xi$ would hence involve modelling of $\tau$ by a subgrid model $m(\bar{u})$ and evaluation of the numerical high-pass filter acting on $\bar{u}^{2}$.

180 The difference between $\Xi$ for a given discretisation method and $\tau$ depends on the subgrid resolution $r=\Delta / h$, which characterises the strength of the numerical filtering. If the subgrid resolution $r$ is sufficiently large, the numerical filter operator $\widehat{(\cdot)}$ approaches the identity operator for all length scales relevant to $\tau$. Hence, as follows from (9) this implies that $\Xi \rightarrow \tau$, and consequently (8) reduces to the filtered inviscid Burgers equation. In practical situations, 185 the grid spacing is chosen such that $r$ assumes quite modest values, and the numerical filter component in the full closure problem needs to be explicitly accounted for. The influence of the spatial discretisation is often referred to as 'implicit filtering' in the literature (e.g., [30-34]).

For central finite difference schemes, denoted by $\delta^{(n)}$, the induced filter may readily be 190 inferred in the following form:

$$
\delta_{x}^{(n)} f(x)=\sum_{j=1}^{n} \frac{d_{j}}{2 j h}(f(x+j h)-f(x-j h))=\partial_{x}\left(\mathcal{L}^{(n)}(f)\right),
$$

where $\mathcal{L}^{(n)}$ denotes the induced filter and $\left\{d_{j}\right\}$ are the differencing weights. For convenience we restrict ourselves to uniform grids. The filter $\mathcal{L}^{(n)}$ may be expressed in terms of a weighted average involving the top-hat filter. This is well known in numerical literature and is directly related to Richardson extrapolation, which may be used to formulate higher-order methods in 195 terms of a judicious combination of lower-order methods. As an example, for the second-order central discretisation, we have

$$
\delta_{x}^{(1)} f(x)=\frac{1}{2 h}(f(x+h)-f(x-h)),
$$

which corresponds to $n=1$ and $d_{1}=1$. Hence, we find

$$
\delta_{x}^{(1)} f(x)=\partial_{x}\left(\int_{x-h}^{x+h} \frac{f(s)}{2 h} \mathrm{~d} s\right)=\partial_{x}(\ell(f, x:-h, h))=\partial_{x}\left(\mathcal{L}^{(1)}(f)\right) .
$$


The induced filter $\mathcal{L}^{(1)}(f)=\ell(f, x:-h, h)$ in terms of the local top-hat filter

$$
\bar{u}=\ell(u, x: a, b)=\int_{x+a}^{x+b} \frac{u(s)}{b-a} \mathrm{~d} s
$$

for which $\Delta=b-a$. Hence, we observe that the second-order central differencing method induces a spatial top-hat filter with filter width equal to twice the grid spacing $h$. The fourth- 200 order accurate central differencing method corresponds to $n=2$ and may be written as

$$
\begin{aligned}
\delta_{x}^{(2)} f(x) & =\frac{1}{12 h}(-f(x+2 h)+8 f(x+h)-8 f(x-h)+f(x-2 h)) \\
& =\frac{4}{3}\left(\frac{f(x+h)-f(x-h)}{2 h}\right)-\frac{1}{3}\left(\frac{f(x+2 h)-f(x-2 h)}{4 h}\right),
\end{aligned}
$$

from which we infer that $d_{1}=4 / 3$ and $d_{2}=-1 / 3$. Hence, the induced filter $\mathcal{L}^{(2)}$ corresponding to $\delta_{x}^{(2)} f(x)$ may be written as

$$
\delta_{x}^{(2)} f(x)=\partial_{x}\left(\frac{4}{3} \ell(f, x:-h, h)-\frac{1}{3} \ell(f, x:-2 h, 2 h)\right)=\partial_{x}\left(\mathcal{L}^{(2)}(f)\right) .
$$

A less well-known example in this sequence is the sixth-order discretisation scheme. This corresponds to $n=3$ and may be written as

$$
\begin{aligned}
\delta_{x}^{(3)} f(x)= & \frac{1}{60 h}(f(x+3 h)-9 f(x+2 h)+45 f(x+h)-45 f(x-h)+9 f(x-2 h) \\
& -f(x-3 h)) \\
= & \frac{3}{2}\left(\frac{f(x+h)-f(x-h)}{2 h}\right)-\frac{3}{5}\left(\frac{f(x+2 h)-f(x-2 h)}{4 h}\right) \\
& +\frac{1}{10}\left(\frac{f(x+3 h)-f(x-3 h)}{6 h}\right) \\
= & \partial_{x}\left(\frac{3}{2} \ell(f, x:-h, h)-\frac{3}{5} \ell(f, x:-2 h, 2 h)+\frac{1}{10} \ell(f, x:-3 h, 3 h)\right),
\end{aligned}
$$

from which we infer that $d_{1}=3 / 2, d_{2}=-3 / 5$ and $d_{3}=1 / 10$.

To characterise the numerically induced filter kernels, we may evaluate their effective filter 205 widths [20]. The corresponding effective filter widths are found to be $\Delta^{(1)} / h=2, \Delta^{(2)} / h=$ $36 / 25=1.44$ and $\Delta^{(3)} / h=300 / 239 \approx 1.2552 \ldots$, respectively. We observe that an increase in the order of the finite differencing scheme implies a decrease in the effective filter width of the induced filter. For comparably large flow structures relative to the filter width $\Delta^{(n)}$, this implies that the induced filtering effect will be uniformly diminished with increasing order of the discretisation method. The effect on small-scale structures cannot be fully characterised by $\Delta^{(n)}$ alone. Instead, the behaviour of the Fourier transform of the induced filter kernels is required, to which we turn next.

The effect of the induced numerical filter may be concisely illustrated through its operation on $u=\sin (k x)$. Specifically we may write $\mathcal{L}^{(n)}(u)=\mathcal{G}^{(n)}(k h) u$. For the second-, fourth- and 215 sixth-order finite differencing methods, we find

$$
\begin{aligned}
\mathcal{G}^{(1)}(k h) & =\Gamma(k h) \\
\mathcal{G}^{(2)}(k h) & =\frac{4}{3} \Gamma(k h)-\frac{1}{3} \Gamma(2 k h) \\
\mathcal{G}^{(3)}(k h) & =\frac{3}{2} \Gamma(k h)-\frac{3}{5} \Gamma(2 k h)+\frac{1}{10} \Gamma(3 k h),
\end{aligned}
$$




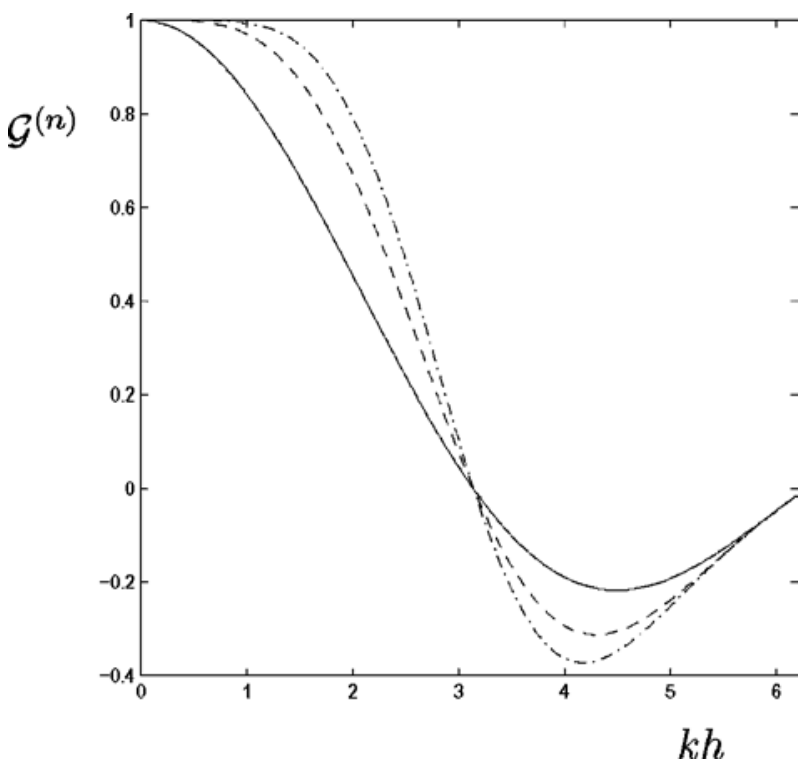

Figure 1. The Fourier transform $\mathcal{G}^{(n)}$ of $2 n$ th-order central discretisation with $n=1$ (solid), $n=2$ (dashed) and $n=3$ (dash-dotted).

where $\Gamma(z)=\sin (z) / z$ is the Fourier transform of the kernel of the top-hat filter. These functions are plotted in figure 1 as a function of $k h$. We note that the filter corresponding to a higher-order spatial discretisation is itself a higher-order filter; with increasing order the

220 Fourier transform of the kernel is seen to stay closer to the identity operator for a wider range of wavenumbers $[20,35,36]$. Each discretisation method gives rise to a particular damping of the amplitude of individual modes, which induces a specific dynamic contribution in an actual large-eddy simulation based on this method. The role of the total filter is discussed further in, e.g., $[35,36]$.

225 To quantify the dynamic effects of the induced filter in more detail, we compare the highpass filter contribution with the explicit closure term in (9). For a large-eddy top-hat filter with filter width $\Delta$, we obtain for $u=\sin (k x)$

$$
\begin{gathered}
\partial_{x} \tau=-k\left(\Gamma^{2}(k \Delta / 2)-\Gamma(k \Delta)\right) \sin (2 k x)=-A_{\tau} \sin (2 k x) \\
\partial_{x}\left(\mathcal{H}^{\bigcap}\left(\bar{u}^{2}\right)\right)=k \Gamma^{2}(k \Delta / 2)\left(1-\mathcal{G}^{(n)}\left(2 k \frac{\Delta}{r}\right)\right) \sin (2 k x)=A_{\mathcal{H}} \sin (2 k x),
\end{gathered}
$$

where we used the sub-filter resolution $r=\Delta / h$ to express the amplitudes entirely as functions of $k \Delta$. The dynamic importance of these terms is represented most directly by considering their amplitudes as a function of $k \Delta$ in figure 2. The magnitudes of these terms depend strongly on the subgrid resolution. As $r=1$ we note that for a wide range of wavenumbers the amplitude of the high-pass filtered contribution is larger than that of the flux due to the turbulent stress. If the subgrid resolution is increased to $r=2$, the numerical high-pass filtered terms decrease considerably and become about equal to or smaller than the turbulent stress flux. Only if we

235 increase to $r=4$, we note that the high-pass filtered contribution is considerably smaller than the turbulent stress flux. This corresponds to an approximately grid-independent large-eddy simulation and the value $r=4$ is consistent with the simulation findings reported in $[10,11]$. The explicit incorporation of the numerical filter effect into the subgrid modelling is the subject of ongoing research and will be published in due course. 


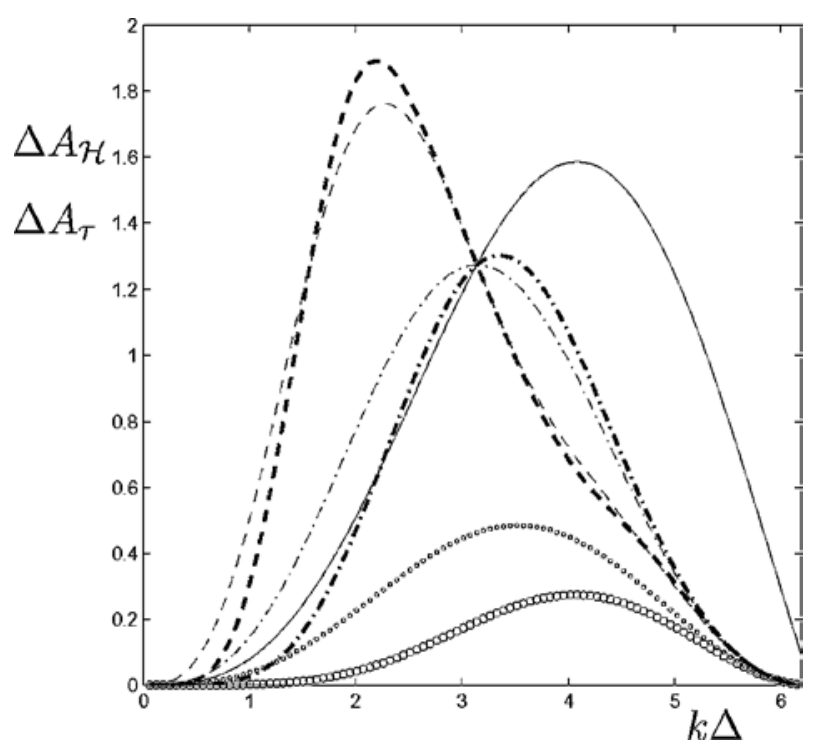

Figure 2. The product of the filter width $\Delta$ and the amplitude of the fluxes due to the turbulent stress tensor $\tau$ (solid) and the high-pass filtered $\mathcal{H}^{(`)}\left(\bar{u}^{2}\right)$ for $r=1$ (dashed), $r=2$ (dash-dotted) and $r=4(0)$ and second-order (thin lines) or fourth-order (thick lines) spatial discretisation.

In the next section we investigate in some detail the total simulation error arising in ac- 240 tual large-eddy simulations and propose a new iterative procedure with which an optimal Smagorinsky constant at a given spatial resolution $h$ may be approximated.

\section{Interacting errors and iterative model optimisation}

In this section we first review the database approach in subsection 3.1. This framework for presenting an overview of the total simulation errors was recently adopted to assess simulation errors in homogeneous turbulence [11]. We illustrate the effect of partial error cancellation. To characterise the interacting errors, we introduce the error landscape associated with the Smagorinsky model and identify the optimal refinement strategy. The optimal refinement strategy also allows as to interpret the degree of optimality of the popular dynamic eddyviscosity model. This subgrid model is shown to display too high levels of eddy-viscosity and 250 yields significant errors at coarse resolutions. Then, in subsection 3.2, we propose a practical iterative procedure to further improve the eddy-viscosity predictions at such coarse resolutions. The new iterative method is based on determining the minimum of the total simulation error. This procedure allows us to efficiently approximate the optimal Smagorinsky constant at a particular resolution and leads to a significant error reduction compared to the dynamic eddy-viscosity predictions.

\subsection{Database approach to interacting errors}

The database approach provides an 'experimental' quantification of the errors that arise in a large-eddy simulation. In particular, it allows a detailed decomposition of the total error in a numerical and a subgrid modelling contribution. This clarifies which effects form the dominant 260 limitations for the overall accuracy of large-eddy simulations, and under what computational 
settings and flow conditions this identification applies. An extensive database of homogeneous decaying turbulence was generated at a variety of resolutions and filter widths using grids with $N^{3}$ grid cells where $N \leq 128$. Initial conditions at Taylor Reynolds numbers $R e_{\lambda}=50$ and

265100 were adopted. For the direct simulation up to $384^{3}$ grid cells were used and the level of convergence for various flow quantities was established [11].

In terms of an explicit filter, it is straightforward to define the different error contributions in a large-eddy simulation [14]. We restrict ourselves to the resolved kinetic energy $E(t)=$ $\left\langle\bar{u}_{i} \bar{u}_{i}\right\rangle / 2$ corresponding to a fixed filter width $\Delta$ and the top-hat filter. The total error in $E$

270 resulting from a 'Smagorinsky fluid' with parameter $\ell_{\mathrm{S}}$ and grid spacing $h=1 / N$ is defined as

$$
e_{\mathrm{tot}}\left(\ell_{\mathrm{S}}, N, t\right)=E_{\overline{\mathrm{DNS}}}(t)-E_{\mathrm{LES}}\left(\ell_{S}, N, t\right) .
$$

We denoted the kinetic energy obtained from filtering the reference direct numerical simulation by $E_{\overline{\mathrm{DNS}}}$. In the following we introduce the central parameter $\xi_{\mathrm{S}}=\ell_{\mathrm{S}} / h$ to represent the resolution of the Smagorinsky length scale. The error $e_{\text {tot }}$ can be further decomposed into a

275 contribution due to the discretisation and the subgrid modelling [14], i.e. $e_{\mathrm{tot}}=e_{\mathrm{d}}+e_{\mathrm{m}}$. To quantify these measures for the simulation error, a DNS and several LES at different $\ell_{\mathrm{S}}$ and spatial resolutions are required, which is at the heart of the database approach. Specifically, we introduce modelling and discretisation errors through

$$
\begin{aligned}
& e_{m}=E_{\overline{\mathrm{DNS}}}(t)-E_{\mathrm{LES}}\left(\ell_{\mathrm{S}}, \infty, t\right) \\
& e_{d}=E_{\mathrm{LES}}\left(\ell_{\mathrm{S}}, \infty, t\right)-E_{\mathrm{LES}}\left(\ell_{\mathrm{S}}, N, t\right) .
\end{aligned}
$$

In these expressions $E_{\mathrm{LES}}\left(\ell_{\mathrm{S}}, \infty, t\right)$ denotes the grid-independent prediction of the decay of resolved kinetic energy obtained with the Smagorinsky model at a fixed Smagorinsky length $\ell_{\mathrm{S}}=C_{\mathrm{S}} \Delta$ and infinite spatial resolution. This grid-independent LES prediction provides a central point of reference in the error decomposition, next to the reference DNS data. It is well approximated at resolutions where $\Delta / h=4-6$. The total error in (22) is time dependent. In order to arrive at a single number to characterise the error in any specific large-eddy simulation,

285 we introduce [10]

$$
\delta_{\mathrm{E}}\left(\xi_{\mathrm{S}}, N\right)=\frac{\left\|e_{\mathrm{tot}}\right\|}{\left\|E_{\overline{\mathrm{DNS}}}\right\|} ; \quad\|f\|^{2}=\frac{1}{T} \int_{0}^{T} f^{2}(s) \mathrm{d} s
$$

with $T$ the total simulation time. A similar procedure may be followed to arrive at $\delta_{\mathrm{E}}^{\mathrm{m}}$ and $\delta_{\mathrm{E}}^{\mathrm{d}}$ for measuring the modelling and discretisation errors. In particular, $e_{\mathrm{tot}}$ in (25) needs to be replaced by $e_{\mathrm{m}}$ or $e_{\mathrm{d}}$, respectively.

We describe the error behaviour in terms of the 'error landscape' that was introduced in

290 [11]. For the Smagorinsky fluid, the error landscape can be expressed using the resolution of the Smagorinsky length $\xi_{\mathrm{S}}=\ell_{\mathrm{S}} / h$ and the spatial resolution $N$ as basic parameters. The error landscape is formed by contours of $\delta_{\mathrm{E}}$ and arises from systematic variation of $\left(\xi_{\mathrm{S}}, N\right)$. It provides the 'optimal refinement strategy' $\widehat{\xi}_{\mathrm{S}}(N)$ which identifies the optimal value of $\ell_{\mathrm{S}}$ that leads to the smallest total error at a particular resolution.

295 The reference direct simulation data and the grid-independent large-eddy predictions allow us to fully separate the modelling and discretisation contributions to the total error. In figure 3 , an error decomposition is collected in which we consider simulations at constant $\ell_{\mathrm{S}}=C_{\mathrm{S}} \Delta$ and various resolutions. In this case we observe, quite paradoxically, that a higher spatial resolution yields results with a larger total error. In fact, the discretisation error effect decreases with increasing resolution and the total error approaches the modelling error. However, this modelling error is by itself larger than the total error on coarser grids. This arises because on 


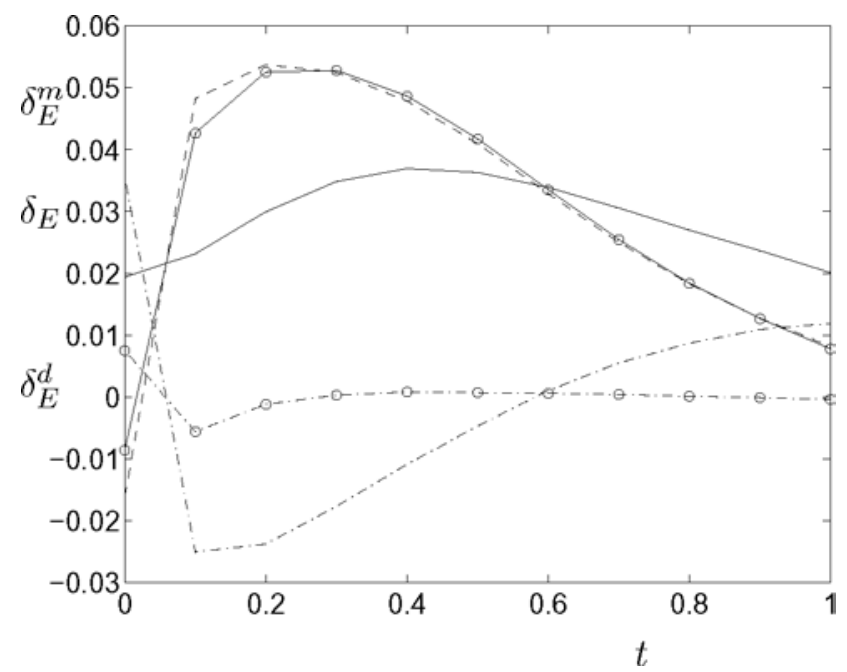

Figure 3. Error decomposition at $\ell_{\mathrm{S}}=6.25 \times 10^{-3}$ for $32^{3}$ (no symbol) and $64^{3}$ (o) displaying total error $\delta_{\mathrm{E}}(-$ ), modelling error $\delta_{\mathrm{E}}^{\mathrm{m}}(--)$, and discretisation error $\delta_{\mathrm{E}}^{\mathrm{d}}(-\cdot)$.

coarse grids the comparably large discretisation error effects partially cancel the modelling error effects.

Collecting the total errors as they arise at various $\left(\xi_{\mathrm{S}}, N\right)$ produces an overview as shown in the error landscape in figure 4 . Contour plots of $\delta_{\mathrm{E}}\left(\xi_{\mathrm{S}}, N\right)$ constitute the error landscape at 305 $R e_{\lambda}=100$, from which the 'optimal refinement strategy' $\widehat{\xi}_{\mathrm{S}}(N)$ can be determined straightforwardly. At coarse resolutions, we observe a fairly sharp increase in the total simulation error in case the Smagorinsky parameter is below the optimal trajectory, while a slightly more gradual increase is observed in case $\ell_{\mathrm{S}}$ is larger than optimal.

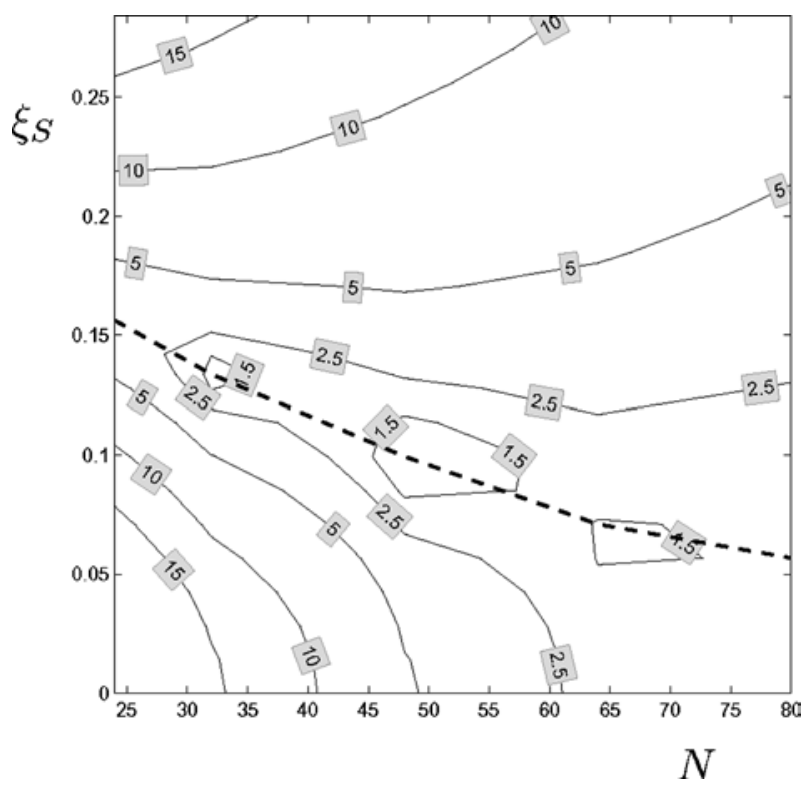

Figure 4. Error landscape based on $\delta_{\mathrm{E}}$ for $R e_{\lambda}=100$. The label on the contours refers to $\delta_{\mathrm{E}}$ in per cent. The thick dashed line corresponds to the optimal refinement strategy. 
310 Optimality of the dynamic procedure. The errors associated with the dynamic procedure may readily be interpreted in relation to the error landscape; the dynamic procedure gives rise to a self-induced length scale $\ell_{\mathrm{d}}(N)$, which is readily translated into a 'dynamic refinement trajectory' $\xi_{\mathrm{d}}(N)=\ell_{\mathrm{d}}(N) / h$ on the $\xi_{\mathrm{S}}-N$ plane. Of particular importance is how this 'dynamic trajectory' $\xi_{\mathrm{d}}(N)$ relates to the 'optimal refinement strategy' $\xi_{\mathrm{S}}(N)$, i.e. does the dynamic

315 procedure approximate the optimal error line? The general trend in the dynamic length scale resolution $\xi_{\mathrm{d}}$ was found to be similar to $\widehat{\xi}_{\mathrm{S}}$, although $\xi_{\mathrm{d}}$ is considerably larger than the optimal value $\widehat{\xi}_{\mathrm{S}}$ [19]. In fact, the errors along the dynamic refinement trajectories are about twice as large as the errors along the optimal refinement trajectory. An effective error reduction arises from grid refinement. Particularly at higher resolutions, a high rate of error reduction with increased resolution is observed (further details may be found in [19]). Conversely, at coarse resolutions the errors are still quite considerable and further improvement would be desired.

\subsection{Iterative approximation of the optimal Smagorinsky constant}

The total simulation error at optimal model parameters can be considerably lower than that which may be obtained with the self-contained dynamic eddy-viscosity model. Especially at low resolution the induced error associated with the dynamic procedure is still quite large. This motivates an alternative optimisation of the Smagorinsky constant at a given spatial resolution, which we introduce in this subsection.

To approximate the lowest total simulation error for the Smagorinsky fluid at a given spatial resolution $N^{3}$, we may adapt the Smagorinsky constant iteratively. The first task is to obtain an interval $[a, c]$ which contains the optimal value for $\xi_{\mathrm{S}}$. Subsequently, this interval will iteratively be reduced in size until an acceptable approximation of the minimal simulation error is achieved. As points of reference, we start from the 'no-model' simulation which corresponds to $\xi_{\mathrm{S}}=0=a$. This is the first simulation that is required in our iterative approach and characterises the effects of the discretisation error only. A second point of reference is obtained at fairly large $\xi_{\mathrm{S}}$. A 'practical upper bound' for $\xi_{\mathrm{S}}$ may be obtained by taking $\xi_{\mathrm{S}}=c=\xi_{d}(N)$. This requires a second large-eddy simulation, now based on the dynamic eddy-viscosity model. Note that the dynamic eddy-viscosity model is used only to provide an upper-bound estimate; all other simulations employ the Smagorinsky model. The optimum is now bracketed by $[a, c]$.

340 In order to start the process of successive approximation of the optimal value for $\xi_{\mathrm{S}}$ at the given resolution $N^{3}$, we evaluate the total error at an interior point of $[a, c]$. For this, we may select the mid-point $\xi_{\mathrm{S}}=b=(a+c) / 2=c / 2$. The simulation at $\xi_{\mathrm{S}}=b$ is the third large-eddy simulation in our iterative approach. The total simulation error $\delta_{\mathrm{E}}\left(\xi_{\mathrm{S}}\right)$ is now such that $\delta_{\mathrm{E}}(a) \geq \delta_{\mathrm{E}}(b)$ and $\delta_{\mathrm{E}}(b) \leq \delta_{\mathrm{E}}(c)$ (note that here and in the following we drop the explicit

345 dependence on $N$ from the notation for convenience, as this parameter is kept constant in the iterations).

Further improvements in $\xi_{\mathrm{S}}$ may be obtained iteratively. In view of the high computational effort that is required to evaluate the 'cost function' $\delta_{\mathrm{E}}\left(\xi_{\mathrm{S}}\right)$, only minimisation algorithms that do not rely on the explicit use of derivatives of $\delta_{\mathrm{E}}$ will be considered [37]. Locally around its minimum we assume that the cost function may be approximated by a parabola. This motivates the use of SIPI to obtain a next estimate for $\xi_{\mathrm{S}}$. Referring to figure 5, we start by constructing an interpolating parabola through the original bracketing triplet, $\left(a, \delta_{\mathrm{E}}(a)\right)$, $\left(b, \delta_{\mathrm{E}}(b)\right)$ and $\left(c, \delta_{\mathrm{E}}(c)\right)$. The location of the minimum of this parabola is at

$$
d=b-\frac{1}{2} \frac{(b-a)^{2}\left[\delta_{\mathrm{E}}(b)-\delta_{\mathrm{E}}(c)\right]-(b-c)^{2}\left[\delta_{\mathrm{E}}(b)-\delta_{\mathrm{E}}(a)\right]}{(b-a)\left[\delta_{\mathrm{E}}(b)-\delta_{\mathrm{E}}(c)\right]-(b-c)\left[\delta_{\mathrm{E}}(b)-\delta_{\mathrm{E}}(a)\right]},
$$




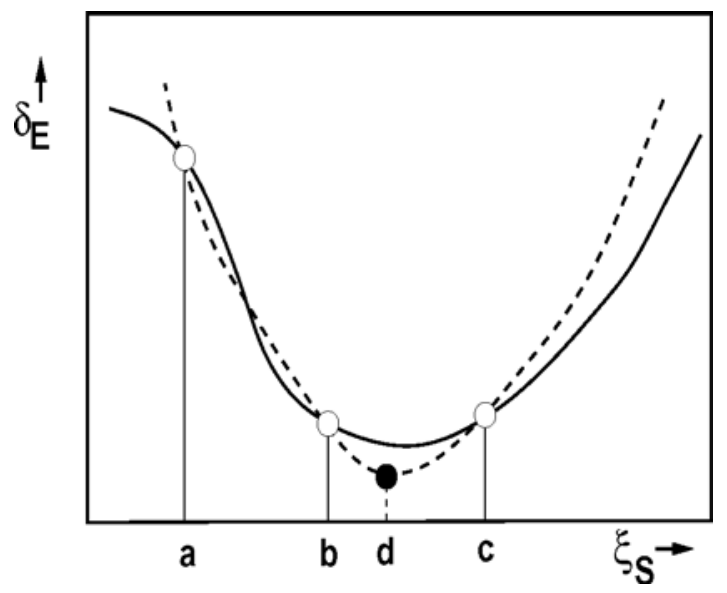

Figure 5. Illustration of successive inverse parabolic interpolation (SIPI) to approximate the optimal resolution of the Smagorinsky length scale $\xi_{\mathrm{S}}=\left(C_{\mathrm{S}} \Delta\right) / h$. The initial triplet $(a, b, c)$ defines an interpolating polynomial (dashed), whose minimum yields a next approximation $d$ at which a new large-eddy simulation should be performed.

provided the three points are not collinear. Subsequently, the total simulation error is evaluated 355 at $\xi_{\mathrm{S}}=d$. From this information, a new bracketing triplet may be identified which defines a new interpolating polynomial, and the process may be continued. Successive inverse parabolic interpolation and evaluation of $\delta_{\mathrm{E}}$ leads to a sequence of bracketing triplets which quite rapidly converges to the optimum. If $\delta_{\mathrm{E}}$ has a continuous second derivative which is positive at the minimum, then the convergence is super-linear.

The application of this method to the Smagorinsky fluid at $R e=100$ and a spatial resolution of $N=32^{3}$ or $N=48^{3}$ is illustrated in figure 6 . In these cases the combination of a 'no-model

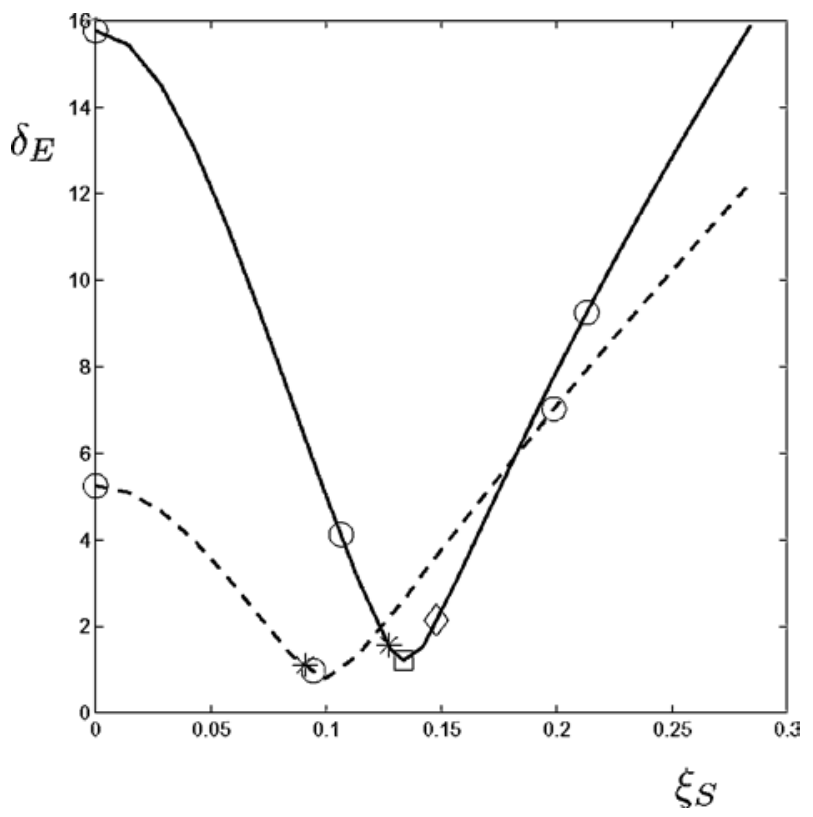

Figure 6. Application of SIPI to the total simulation error at $R e_{\lambda}=100$ and $N=32$ (solid) and $N=48$ (dashed). The initial triplet is indicated with $\circ$, the first iterand with $*$, the second with $\diamond$ and the third with $\square$. 
Table 1. Iterands approximating the optimal simulation setting corresponding to $R e_{\lambda}=100$ at $N=32^{3}$ and $N=48^{3}$. The values $\xi_{\mathrm{S}}=0.213$ and $\xi_{\mathrm{S}}=0.1988$ correspond to $\xi_{d}$ obtained with the dynamic procedure at these resolutions.

\begin{tabular}{lrlc}
\hline$\xi_{\mathrm{S}}$ & $\delta_{\mathrm{E}}$ at $N=32^{3}(\%)$ & \multicolumn{1}{c}{$\xi_{\mathrm{S}}$} & $\delta_{\mathrm{E}}$ at $N=48^{3}(\%)$ \\
\hline 0 & 15.77 & 0 & 5.249 \\
0.213 & 9.24 & 0.1988 & 7.015 \\
0.1065 & 4.12 & 0.0944 & 0.971 \\
0.1272 & 1.56 & 0.09084 & 1.100 \\
0.1477 & 2.14 & & \\
0.1336 & 1.21 & & \\
\hline
\end{tabular}

simulation', a 'dynamic eddy-viscosity simulation' and a Smagorinsky simulation with $\xi_{\mathrm{S}}=$ $\xi_{d} / 2$ already yields about a factor of 4 reduction of the error relative to the no-model case.

365 The subsequent application of SIPI yields a rapid, albeit non-uniform, convergence as may be inferred from table 1 . After about four to six properly selected large-eddy simulations, the optimum is quite well approximated and a relative error of about $1-2 \%$ remains.

The computational overhead of the additional large-eddy simulations required for the iterations is well justified. This may be quantified by incorporating the scaling of CPU time with resolution, i.e. $\sim N^{4}$. In fact, compared to the costs at $N=32^{3}$, a simulation at $N=48^{3}$ is about $(48 / 32)^{4}=5.0625$ times more expensive and using $N=64^{3}$ requires $2^{4}=16$ times more effort [10]. In this case, the approximate optimisation of $\xi_{\mathrm{S}}$ at $N=32^{3}$ was seen to require about four large-eddy simulations, which can be completed within the cost of one additional simulation at $N=48^{3}$. The difference in computational costs compared to the higher

375 resolution cases is even more striking. Hence, the iterative improvement of $\xi_{\mathrm{S}}$ at $N=32^{3} \mathrm{com}$ pares very favourably with the non-optimised dynamic eddy-viscosity simulations at higher resolutions. This may readily be inferred from the error levels along the dynamic refinement trajectories which yield about $7 \%$ and $4.5 \%$ for $N=48^{3}$ and $N=64^{3}$, respectively [19]. However, the accuracy at $N=32^{3}$ can never be better than the optimum of about $1 \%$. If higher accuracy is needed, then one should resort to higher resolutions.

The optimisation procedure described here provides a very simple illustration of the consequences of interacting errors. The error reduction relies specifically on the observed structure of the error landscape. Although the search procedure for an optimum adds significantly to the total computational cost, the reduction in the error well justifies this overhead. Further developments are required to render this approach suitable for more complex applications.

Central to an optimisation procedure is the formulation of the 'cost function' to quantify the errors that occur. Here, we compared LES to available DNS data, this constitutes a rather academic setting. Moreover, we only considered deviations with respect to one flow property. In general, one frequently encounters situations in which the accurate prediction of a collection of 'monitoring quantities' is desired. This requires simultaneous accuracy for each of these quantities and implies an appropriate weighing of individual errors as part of the total error measure. The total cost function may also incorporate possibly available experimental data or theoretical predictions to express the degree of error in a specific simulation. The extension of the SIPI optimisation procedure to spatially inhomogeneous turbulent flows is another matter of interest. Depending on the particular application, a zonal approach might be considered in which one optimises an error measure that is composed of contributions from various regions in the flow domain. Once a cost function has been defined, model parameters may be iteratively adapted following SIPI. The solution can be systematically improved, relative to the adopted cost function. Of course, the optimum that is obtained is intimately related to this cost function; this may be application specific and requires further attention. 


\section{Concluding remarks}

In this paper we considered the modifications of the large-eddy closure problem arising from the spatial discretisation at coarse subgrid resolutions. Moreover, a new iterative method for approximating the optimal Smagorinsky constant at low resolutions was proposed and applied to homogeneous, decaying turbulence.

In case the sub-filter resolution $r=\Delta / h$ is low, the particular discretisation scheme that is adopted in the computational model was shown to have a large dynamic effect relative to the flux due to the turbulent stress. The difference between the actual computational stress tensor $\Xi$ and the turbulent stress tensor $\tau$ may be expressed most directly in terms of the modified equation. An additional flux arises in the modified equation which incorporates the numerical high-pass filter applied to $\bar{u}^{2}$. The analysis suggests that considerable improvements of practical large-eddy simulation may be achieved in case the closure of the modified equation is made to explicitly account for the coarseness as well as the type of discretisation.

The induced numerical filter corresponding to central finite differencing was explicitly calculated. It was shown that higher-order discretisation schemes induce higher-order filters that may be expressed as linear combinations of the top-hat filter. The dynamic importance of the high-pass filter contributions relative to the turbulent stress tensor depends considerably on the sub-filter resolution. For values as low as $r=1-2$, the induced high-pass filter contribution is comparable to or even larger than the term that requires closure in a large-eddy simulation. This was observed earlier in a posteriori analysis of turbulent mixing [14]. In case $r \geq 4$, it appears that the dynamic consequences of the high-pass filter term can safely be neglected. The reduction of the numerical influences with increasing $r$ is stronger in case the order of accuracy of the spatial discretisation is higher. This underlines and further quantifies the observations reported in $[10,11]$.

For an explicit time-stepping method and a uniform computational grid, every doubling of the sub-filter resolution $r$ at fixed filter width $\Delta$ corresponds to about a factor of 16 increase in a computational effort [38]. This makes the near-grid-independence requirement $r \geq 4$ quite unattainable in practical applications of large-eddy simulation. To investigate the error dynamics at coarser resolutions, a database approach may be followed. This was first considered for homogeneous decaying turbulence in [11]. This method provides a detailed view of the intricate error interactions in large-eddy simulation.

The basic modelling and discretisation errors were found to counteract. This leads to an intriguing paradox related to possible strategies that should be followed to further improve large-eddy predictions compared to some reference simulation. While it is tempt- 435 ing to think that a higher resolution, a better numerical method or a more precise subgrid model would always lead to an improved accuracy of the predictions, the counteracting property of the errors and their specific reverse dependence on filter width can completely distort this impression. Rather, the total error arises from a balance between modelling and discretisation errors and it is not an easy matter to predict a priori whether these errors will or will not counteract and what the magnitude of the individual error contributions is.

The optimal working conditions for large-eddy simulations may be inferred from an error landscape. The use of optimal refinement strategies [11] as a point of reference for the evaluation of the dynamic procedure was reviewed. The dynamic procedure was found to provide a 445 build-in 'dynamic trajectory', which follows the main Reynolds number and resolution trends seen in the 'optimal refinement strategy' relatively well. However, an over-prediction of the optimal resolution of the Smagorinsky length scale is obtained, which was found to lead to errors about twice as high as the optimal errors. 
450 To improve upon these shortcomings of the popular dynamic eddy-viscosity model, a new iterative optimisation procedure for the Smagorinsky constant was proposed. This procedure is based on successive inverse polynomial interpolation (SIPI) and yields strongly improved accuracy compared to predictions based on the dynamic eddy-viscosity model. Initially, this method requires a bracketing interval for which simulations without a subgrid model and with

455 the dynamic eddy-viscosity model were adopted. About two to four additional large-eddy simulations were found to yield a near-optimal value of $\xi_{\mathrm{S}}$ for the case $N=32^{3}$ at a Taylor Reynolds number $R e_{\lambda}=100$. The large computational overhead associated with this iterative procedure is well justified in view of the increased accuracy compared to the dynamic eddyviscosity model. Without the optimisation, such an error level would require much higher resolutions and computational costs. Further analysis of this new iterative procedure is the subject of ongoing research.

\section{Acknowledgements}

The author gratefully acknowledges stimulating discussions with Johan Meyers (KU Leuven) and Jochen Fröhlich (Karlsruhe). Simulations were performed at SARA and made possible through grant SG-213 of the Dutch National Computing Foundation (NCF).

\section{References}

[1] Geurts, B.J., 2003, Elements of Direct and Large Eddy Simulation. Edwards Publishing.

[2] Meneveau, C. and Katz, J., 2000, Scale-invariance and turbulence models for large eddy simulation. Annual Review of Fluid Mechanics, 32, 1.

470 [3] Lesieur, M. and Metais, O., 1996, New trends in large-eddy simulations of turbulence. Annual Review of Fluid Mechanics, 28, 45.

[4] Sagaut, P., 2001, Large eddy simulation for incompressible flows: an introduction. Scientific Computation (Verlag: Springer).

[5] Sarghini, F., Piomelli, U. and Balaras, E., 1999, Scale-similar models for large-eddy simulations. Physics of Fluids, 11, 1596

[6] Keating, A., Piomelli, U., Balaras, E. and Kaltenbachj, H.-J., 2004, A priori and a posteriori tests of inflow conditions for large-eddy simulation. Physics of Fluids, 16, 4696.

[7] Park, N., Yoo, J.Y. and Choi, H., 2005, Toward improved consistency of a priori tests with a posteriori tests in large eddy simulation. Physics of Fluids, 17, 015103.

480 [8] Rogallo, R.S. and Moin, P., 1984, Numerical simulation of turbulent flows. Annual Review of Fluid Mechanics, 16, 99 .

[9] Salvetti, M.V. and Beux, F., 1998, The effect of the numerical scheme on the subgrid scale term in large-eddy simulation. Physics of Fluids, 10, 3020.

[10] Geurts, B.J. and Fröhlich, J., 2002, A framework for predicting accuracy limitations in large eddy simulation. Physics of Fluids, 14, L41.

[11] Meyers, J., Geurts, B.J. and Baelmans, M., 2003, Database analysis of errors in large eddy simulation. Physics of Fluids, 15, 2740.

[12] Ghosal, S., 1996. An analysis of numerical errors in large-eddy simulations of turbulence. Journal of Computational Physics, 125, 187.

490 [13] Kravchenko, A.G. and Moin, P., 1997, On the effect of numerical errors in large eddy simulation of turbulent flows. Journal of Computational Physics, 131, 310.

[14] Vreman, A.W., Geurts, B.J. and Kuerten, J.G.M., 1996, Comparison of numerical schemes in large Eddy simulation of the temporal mixing layer. International Journal for Numerical Methods in Fluids, 22, 299.

[15] Gullbrand, J. and Chow, F.K., 2003, The effect of numerical errors and turbulence models in large-eddy simulation of turbulent channel flow, with and without explicit filtering. Journal of Fluid Mechanics, $495,323$.

[16] Carati, D., Winckelmans, G.S. and Jeanmart H., 2001, On the modelling of the subgrid-scale and filtered-scale stress tensors in large-eddy simulation. Journal of Fluid Mechanics, 441, 119.

[17] Winckelmans, G.S., Wray, A.A., Vasilyev, O.V. and Jeanmart, H., 2001, Explicit filtering large-eddy simulation using the tensor-diffusivity model supplemented by a dynamic Smagorinsky term. Physics of Fluids, 13, 1385$500 \quad 1403$.

[18] Germano, M., Piomelli, U., Moin, P. and Cabot, W.H., 1991, A dynamic subgrid-scale model. Physics of Fluids, 3, 1760 . 
[19] Meyers, J., Geurts, B.J. and Baelmans, M., 2005, Optimality of the dynamic procedure for large-eddy simulation. Physics of Fluids, 17, 045108.

[20] Bos, F. van der and Geurts, B.J., 2005, Commutator errors in the filtering approach to large-eddy simulation. 505 Physics of Fluids, 17, 035108.

[21] Burton, G.C. and Dahm, W.J.A., 2005, Multifractal subgrid-scale modeling for large-eddy simulation: II. Backscatter limiting and a posteriori evaluation. Physics of Fluids, 17, 075112.

[22] Mason, P.J. and Thomson, D.J., 1992, Stochastic backscatter in large-eddy simulations of boundary layers. Journal Fluid Mechanics, 242, 51.

[23] Leith, C.E., 1990, Stochastic backscatter in a subgrid-scale model: plane shear mixing layer. Physics of Fluids, 2, 297.

[24] Smagorinsky, J., 1963, General circulation experiments with the primitive equations. Monthly Weather Review, 91, 99.

[25] Mason, P.J. and Callen, N.S., 1986, On the magnitude of the subgrid scale eddy coefficient in large eddy simulations of turbulent channel flow. Journal of Fluid Mechanics, 162, 439.

[26] Germano, M., 1992, Turbulence: the filtering approach. Journal of Fluid Mechanics, 238, 325.

[27] Lilly, D.K., 1992, A proposed modification of the Germano subgrid-scale closure method. Physics of Fluids, 4, 633 .

[28] Pope, S.B., 2000, Turbulent flows. (Cambridge: Cambridge University Press).

[29] Geurts, B.J. and Bos, F. van der, 2005, Numerically induced high-pass dynamics in large-eddy simulation. Physics of Fluids, 17, 125103.

[30] Lund, T.S. and Kaltenbach, H.J., 1995. Experiments with explicit filtering for LES using a finite-difference methods. CTR Annual Research Briefs, 91.

[31] Lund, T.S., 2003. The use of explicit filters in large eddy simulation. Computers \& Mathematics with Appli- 525 cation, 46, 603.

[32] Boris, J.P., Grinstein, F.F., Oran, E.S. and Kolbe, R.L., 1992. New insights into large-eddy simulation. Fluid Dynamics Research, 10, 199.

[33] Fureby, C. and Grinstein, F.F., 1999, Monotonically integrated large eddy simulation of free shear flows. AIAA Journal, 37, 544.

[34] Adam, N.A., Hickel, S. and Franz, S., 2004, Implicit subgrid-scale modeling by adaptive deconvolution. Journal of Computational Physics, 200, 412.

[35] Geurts, B.J., 1997, Inverse modeling for large-eddy simulation. Physics of Fluids, 9, 3585.

[36] Vasilyev, O.V., Lund, T.S. and Moin, P., 1998, A general class of commutative filters for LES in complex geometries. Journal of Computational Physics, 146, 82-104.

[37] Brent, R., 1973, Algorithms for Minimization without Derivatives. (Englewood Cliffs, NJ: Prentice-Hall).

[38] Geurts, B.J. and Fröhlich, J, 2001. Numerical effects contaminating LES: a mixed story. Modern Strategies for Turbulent Flow Simulation, B.J. Geurts, (Ed) (Edwards Publishing), p 317. 


\section{Queries}

Q1. Au: Pls. provide keywords.

Q2. Au: Please provide the place of the publisher for references [1] and [38].

Q3. Au: Please provide volume no. for reference [30]. 